\title{
MINIMAL DISCRETE ENERGY PROBLEMS AND NUMERICAL INTEGRATION ON COMPACT SETS IN EUCLIDEAN SPACES
}

By

\section{S.B. Damelin}

and

V. Maymeskul

IMA Preprint Series \# 2073

( October 2005)

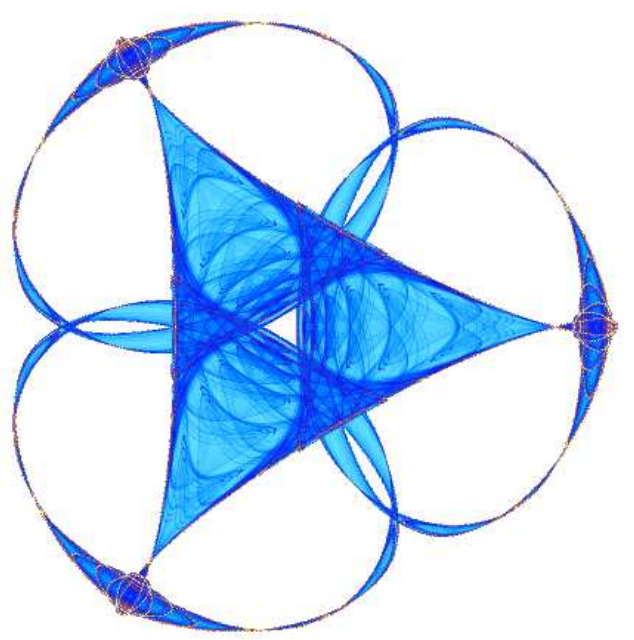

INSTITUTE FOR MATHEMATICS AND ITS APPLICATIONS

UNIVERSITY OF MINNESOTA 400 Lind Hall

207 Church Street S.E.

Minneapolis, Minnesota 55455-0436

Phone: 612/624-6066 Fax: 612/626-7370

URL: http://www.ima.umn.edu 


\title{
Minimal Discrete Energy Problems and Numerical Integration on Compact Sets in Euclidean Spaces
}

\author{
S.B. Damelin* and V. Maymeskul
}

October 25,2005

\begin{abstract}
In this paper, we announce and survey recent results on (1) point energies, scar defects, separation and mesh norm for optimal $N \geq 1$ arrangments of points on a class of $d$-dimensional compact sets embedded in $\mathbb{R}^{n}, n \geq 1$, which interact through a Riesz potential, and (2) discrepancy estimates of numerical integration on the $d$-dimensional unit sphere $S^{d}, d \geq 2$.
\end{abstract}

Keywords and Phrases: Discrepancy, Discrete Riesz Energy, Mesh Norm, Numerical Integration, Point Energies, Potential, Quadrature, Riesz Points, Separation, Sphere.

\section{Table of Contents}

(a) Introduction.

(b) Point Energies, Separation, and Mesh Norm for Optimal Riesz Points on $d$-Rectifiable Sets.

(c) Discrepancy and Errors of Numerical Integration on Spheres.

\section{Introduction}

\subsection{Discrete Riesz Energy Problems}

The problem of uniformly distributing points on spheres (more generally, on compact sets in $\mathbb{R}^{n}$ ) is an interesting and difficult problem. It is folklore, that such problems were discussed already by Carl Friedrich Gauss in his famous Disqvistiones arithmaticae, although it is most likely that similar problems appeared in mathematical writings even before that time.

For $d \geq 1$, let $S^{d}$ denote the $d$-dimensional unit sphere in $\mathbb{R}^{d+1}$, given by

$$
x_{1}^{2}+\cdots+x_{d+1}^{2}=1 .
$$

For $d=1$, the problem is reduced to uniformly distributing $N$ points on a circle, and equidistant points or the vertices of a regular $N$-gon provide an obvious answer. For $d \geq 2$, the problem becomes much more difficult; in fact, there are numerous criteria for uniformity, resulting in different optimal configurations on the sphere. It is of some interest on its own to describe a "well distributed" point set of cardinality $N$ and even to define suitable notions of what "well distributed" should mean. On the other hand, numerical integration procedures on the sphere typically require node sets, which are spread evenly. Numerous constructions of "well-distributed" point sets have been given in the literature. These include constructions of

*This author is supported, in part, by EP/C000285 and NSF-DMS-0439734. 
generalized spiral points, low-discrepancy point sets in the unit cube, which can be transformed via standard parametrizations, constructions given by integer solutions of the equation

$$
x_{1}^{2}+\cdots+x_{d+1}^{2}=N
$$

projected onto the sphere, rotations of certain subgroups applied to points on the sphere, finite field constructions of point sets based on finite field solutions of (2.1), and associated combinatorial designs. See $[2,5,14,15,17]$ and the references cited therein.

In this paper, we are interested in studying certain arrangements of $N$ points on a class of $d$-dimensional compact sets $A$ embedded in $\mathbb{R}^{n}$. We assume that these points interact through a power law (Riesz) potential $V=r^{-s}$, where $s>0$ and $r$ is the Euclidean distance in $\mathbb{R}^{n}$.

Given a compact set $A \subset \mathbb{R}^{n}, s>0$, and a collection $\omega_{N}=\left\{x_{1}, \ldots, x_{N}\right\}$ of distinct points on $A$, the discrete Riesz s-energy associated with $\omega_{N}$ is given by

$$
E_{s}\left(A, \omega_{N}\right):=\sum_{1 \leq i<j \leq N}\left|x_{i}-x_{j}\right|^{-s} .
$$

Let $\omega_{N}^{*}:=\left\{x_{1}^{*}, \ldots, x_{N}^{*}\right\} \subset A$ be a configuration, for which $E_{s}\left(A, \omega_{n}\right)$ attains its minimal value; that is,

$$
\mathcal{E}_{s}(A, N):=\min _{\omega_{N} \subset A} E_{s}\left(A, \omega_{N}\right)=E_{s}\left(A, \omega_{N}^{*}\right) .
$$

In accordance with convention, we shall call such minimizing configurations $s$-extremal configurations. It is well-known that, in general, $s$-extremal configurations are not always unique. For example, in the case of $S^{d}$, they are invariant under rotations. A natural physical interpretation of minimal energy problem on the sphere is the electron problem, which asks for distributions of electrons in stable equilibrium.

Natural questions that arise in studying the discrete Riesz energy are:

(1) What is the asymptotic behavior of $\mathcal{E}_{s}(A, N)$, as $N \rightarrow \infty$ ?

(2) How are $s$-extremal configurations distributed on $A$ for large $N$ ?

It is well-known, that answers to these questions essentially depend on the relation between $s$ and the Hausdorff dimension $d_{H}(A)$ of $A$. We demonstrate this fact with the following two classical examples and refer the reader to $[6,10,12,16]$ and the references cited therein for more details.

Example 1 The interval $[-1,1], d_{H}([-1,1])=1$ : It is known that $s=1$ is the critical value in the sense that $s$-extremal configurations are distributed on $[-1,1]$ differently for $s<1$ and $s \geq 1$. Indeed, for $s<1$, the limiting distribution of $s$-extremal configurations has an arcsine-type density

$$
\mu_{s}^{\prime}(x):=\frac{\Gamma(1+s / 2)}{\sqrt{\pi} \Gamma((1+s) / 2)}\left(1-x^{2}\right)^{(s-1) / 2}
$$

and, for $s \geq 1$, the limiting distribution is the uniform distribution on $[-1,1]([16])$. Concerning the minimal energies, they again behave differently for $s<1, s=1$, and $s>1$. Namely, with $e_{s}:=$ $[\sqrt{\pi} \Gamma(1+s / 2)] /[\cos (\pi s / 2) \Gamma((1+s) / 2)]$,

$$
\mathcal{E}_{s}([-1,1], N) \sim \begin{cases}(1 / 2) N^{2} e_{s}, & s<1, \\ (1 / 2) N^{2} \ln N, & s=1, \\ (1 / 2)^{s} \zeta(s) e(s) N^{1+s}, & s>1,\end{cases}
$$

where $\zeta(s)$ stands for the Riemann zeta function.

The dependence of the distribution of $s$-extremal configurations over $[-1,1]$ and the asymptotics for minimal discrete $s$-energy on $s$ can be explained using potential theory. Indeed, for a probability Borel measure $\nu$ on $[-1,1]$, its $s$-energy integral is defined to be

$$
I_{s}([-1,1], \nu):=\iint_{[-1,1]^{2}}|x-y|^{-s} d \nu(x) d \nu(y),
$$


which can be finite or infinite. For a point set $\omega_{N}=\left\{x_{1}, \ldots, x_{N}\right\}$ on $[-1,1]$, let

$$
\nu^{\omega_{N}}:=\frac{1}{N} \sum_{j=1}^{N} \delta_{x_{j}}
$$

denote the normalized counting measure of $\omega_{N}$ (so that $\left.\nu^{\omega_{N}}([-1,1])=1\right)$. Then the discrete Riesz s-energy (2.2), associated with $\omega_{N}$, can be written as

$$
E_{s}\left([-1,1], \omega_{N}\right)=(1 / 2) N^{2} \iint_{x \neq y}|x-y|^{-s} d \nu^{\omega_{N}}(x) d \nu^{\omega_{N}}(y),
$$

where the integral represents a discrete analog of the $s$-energy integral (2.5) for the point-mass measure $\nu^{\omega_{N}}$.

If $s<1$, then it is well-known that the energy integral (2.5) is minimized uniquely by an arcsine-type measure $\mu_{s}$, whose density with respect to the Lebesgue measure is given by (2.4). On the other hand, the normalized counting measure $\nu_{N}^{\omega_{N}^{*}}$ of an $s$-extremal configuration minimizes the discrete energy integral (2.7) over all configurations $\omega_{N}$ on $[-1,1]$. Thus one can reasonably expect that, for $N$ large, $\nu^{\omega_{N}^{*}}$ is "close" to $\mu_{s}$ and, therefore, the minimal discrete $s$-energy $\mathcal{E}_{s}([-1,1], N)$ is close to $(1 / 2) N^{2} I_{s}\left([-1,1], \mu_{s}\right)=(1 / 2) N^{2} e_{s}$.

If $s \geq 1$, then the energy integral (2.5) diverges for every measure $\nu$. Thus, $\mathcal{E}_{s}([-1,1], N)$ must grow faster than $N^{2}$. Concerning the distribution of $s$-extremal points over $[-1,1]$, the interactions are now strong enough to force them to stay away from each other as far as possible. Of course, depending on $s$, far neighbors still incorporate some energy in $\mathcal{E}_{s}([-1,1], N)$, but the closest neighbors' inputs are dominating. So, $s$-extremal points distribute themselves over $[-1,1]$ in an equally spaced manner.

Example 2 The unit sphere $S^{d}, d_{H}\left(S^{d}\right)=d$ : Here again, there are three cases to consider: $s<d, s=d$, and $s>d$. In all cases, the limiting distribution of $s$-extremal configurations is given by the normalized area measure $\sigma_{d}$ on $S^{d}$, which is natural due to rotation invariance, but the asymptotic behavior of $\mathcal{E}_{s}\left(S^{d}, N\right)$ is quite different (see $[5,9,10,12])$. With

$$
\tau_{s, d}(N):= \begin{cases}N^{2}, & s<d \\ N^{2} \ln N, & s=d \\ N^{1+s / d}, & s>d\end{cases}
$$

it is known that the $\operatorname{limit}_{\lim _{N \rightarrow \infty}} \mathcal{E}_{s}\left(S^{d}, N\right) / \tau_{s, d}(N)$ exists. Moreover, in the first two cases, it has the value $(1 / 2) \gamma_{s, d}$, where

$$
\gamma_{d, d}=\frac{\Gamma((d+1) / 2)}{d \sqrt{\pi} \Gamma(d / 2)} \quad \text { and } \quad \gamma_{s, d}=\frac{\Gamma((d+1) / 2) \Gamma(d-s)}{\Gamma((d-s+1) / 2) \Gamma(d-s / 2)}, \quad s<d .
$$

The dependence of the growth rate of $\mathcal{E}_{s}\left(S^{d}, N\right)$ on $s$ can be explained using potential theory arguments similar to those in Example 1.

In Section 3 we describe some recent results of the authors obtained in $[6,7]$ concerning separation, mesh norm, and point energies of $s$-extremal Riesz configurations on a wide class of compact sets in $\mathbb{R}^{n}$, and refer the reader to some latest results of other authors in this area. In particular, we give new separation estimates for the Riesz points on the unit sphere $S^{d}$ for the case $0<s<d-1$ and confirm scar defects conjecture ([4]) based on numerical experiments.

\section{$2.2 \quad$ Numerical Integration and $g$-Functionals}

Numerical integration and discrepancy estimates are important problems in applied mathematics and many applications, when one needs to approximate the quantity $\sup _{f \in \mathcal{F}}\left|\int_{\mathcal{B}} f d \zeta\right|$, where $\mathcal{B} \subset \mathbb{R}^{n}, n \geq 3$, is a bounded domain or manifold, $\zeta: \mathbb{R}^{n} \rightarrow \mathbb{R}$ is a Borel measure with compact support in $\mathcal{B}$ and $\mathcal{F}$ is a suitable 
class of real valued functions on $\mathcal{B}$. Such problems arise naturally in many areas of growing interest such as mathematical finance, physical geodesy, meteorology, and diverse mathematical areas such as approximation theory, spherical $t$-designs, discrepancy and combinatorics, Monte-Carlo and Quasi-Monte-Carlo methods, finite fields, information based complexity theory, and statistical learning theory.

For a point set $\omega_{N}=\left\{x_{1}, \ldots, x_{N}\right\}$, a natural measure for the quality of its distribution on the sphere $S^{d}$ is the spherical cap discrepancy

$$
D\left(\omega_{N}\right)=\sup _{C \subseteq S^{d}}\left|\sum_{k=1}^{N}\left[\nu^{\omega_{N}}-\sigma_{d}\right](C)\right|,
$$

where the supremum ranges over all spherical caps $C \subseteq S^{d}$ (intersections of half spaces with $S^{d}$ ) and $\nu^{\omega_{N}}$ is the normalized counting measure (2.6) of $\omega_{N}$. The discrepancy simply measures the maximal deviation between $\nu^{\omega_{N}}$ and the normalized area measure $\sigma_{d}$ over all spherical caps.

For a continuous function $f: S^{d} \rightarrow \mathbb{R}$, we denote by

$$
R\left(f, \omega_{N}\right):=\int_{S^{d}} f(x) d \sigma_{d}(x)-\frac{1}{N} \sum_{k=1}^{N} f\left(x_{k}\right)=\int_{S^{d}} f(x) d\left[\sigma_{d}-\nu^{\omega_{N}}\right]
$$

the error in numerical integration on the sphere $S^{d}$ using nodes in $\omega_{N}$.

In Section 4, we briefly discuss spherical cap discrepancy and error estimates for numerical integration on the $d$-dimensional unit sphere $S^{d}$, and refer the interested reader to [5] and the references cited therein for a comprehensive account of this vast and interesting subject. The methods used in [5] are motivated by the discussion on $s$-energy and $s$-extremal Riesz points presented in Section 3. A crucial observation was the possibility of use of $g$-functionals, generalizing classical Riesz and logarithmic functionals, to estimate the second order terms in the expansions of $g$-energies, which yield errors in numerical integration valid for a large class of smooth functions on the sphere.

\section{Point Energies, Separation, and Mesh Norm for Optimal Riesz Points on $d$-Rectifiable Sets}

In this section, we focus on the main results obtained by the authors in $[6,7]$, which are dealing with properties of $s$-extremal Riesz configurations on compact sets in $\mathbb{R}^{n}$, and refer an interested reader to $[3,8,9,10,12,13,16]$ and references cited therein for results of other authors. Our results imply, in particular, low discrepancy of $s$-extremal Riesz configurations on $S^{d}$.

\subsection{The case $s>d$}

We define a class $\mathcal{A}^{d}$ of $d$-dimensional compact sets $A \subset \mathbb{R}^{n}$ for which, in the case $s \geq d$, the asymptotic behavior of $E_{s}(A, N)$, separation and mesh norm estimates, and the limiting distribution of $\omega_{N}^{*}$ (in terms of weak-star convergence of normalized counting measures) over $A$ have been recently obtained.

Definition 1 We say that a set $A$ belongs to the class $\mathcal{A}^{d}$ if, for some $n \geq d, A \subset \mathbb{R}^{n}$ and

(1) $H^{d}(A)>0$ and

(2) $A$ is a finite union of bi-Lipschitz images of compact sets in $\mathbb{R}^{d}$, that is

$$
A=\bigcup_{i=1}^{m} \phi\left(K_{i}\right)
$$

where each $K_{i} \subset \mathbb{R}^{d}$ is compact and $\phi_{i}: K_{i} \rightarrow \mathbb{R}^{n}$ is bi-Lipschitz on $K_{i}, i=1, \ldots, m$. 
Here and throughout, $H^{d}(\cdot)$ means the $d$-dimensional Hausdorff measure in $\mathbb{R}^{n}$.

For a collection $\omega_{N}=\left\{x_{1}, \ldots, x_{N}\right\}$ of distinct points on a set $A \subset \mathbb{R}^{n}$, we denote

$$
\delta\left(A, \omega_{N}\right):=\min _{y \in \omega_{N}} \min _{\substack{x \in \omega_{N} \\ x \neq y}}|x-y|, \quad \rho\left(A, \omega_{N}\right):=\max _{x \in A} \min _{y \in \omega_{N}}|y-x| .
$$

The quantity $\delta\left(A, \omega_{N}\right)$ is called the separation radius and gives the minimal distance between points in $\omega_{N}$, while the mesh norm $\rho\left(A, \omega_{N}\right)$ means the maximal radius of a "cap" $E(x, r)$ (see (3.1)) on $A$, which does not contain points from $\omega_{N}$. These two quantities, $\delta\left(A, \omega_{N}\right)$ and $\rho\left(A, \omega_{N}\right)$, give a good enough description of the distribution of the points in $\omega_{N}$ over the set $A$. We also define the point energies of the points in $\omega_{N}$ by

$$
E_{j, s}\left(A, \omega_{N}\right):=\sum_{\substack{i=1 \\ i \neq j}}^{N}\left|x_{j}-x_{i}\right|^{-s}, \quad j=1, \ldots, N .
$$

The following two results were established in [6].

Theorem 2 Let $A \in \mathcal{A}^{d}$ and $s>d$. Then, for all $1 \leq j \leq N$,

$$
E_{j, s}\left(A, \omega_{N}^{*}\right) \leq C N^{s / d} .
$$

As a consequence, we get the following (best possible) lower estimate on the separation radius for $s$ extremal configurations.

Corollary 3 For $A \in \mathcal{A}^{d}, s>d$, and any s-extremal configuration $\omega_{N}^{*}$ on $A$,

$$
\delta\left(A, \omega_{N}^{*}\right) \geq c N^{-1 / d} .
$$

Under some additional restrictions on a set $A \in \mathcal{A}^{d}$, this estimate was obtained earlier in [10]. We also refer the reader to a recent paper [3], where similar results were proved for a weighted Riesz energy. [6].

Concerning the mesh norm $\rho\left(A, \omega_{n}^{*}\right)$ of $s$-extremal configurations, the following result was also proved in

Let, for $x \in A$ and $r>0, E(x, r)$ denote a "cap" on $A$ with center $x$ and radius $r$; that is,

$$
E(x, r):=\{y \in A:|y-x|<r\} .
$$

Theorem 4 Let $A \in \mathcal{A}^{d}, s>d$, and suppose further that either $m=1$ in Definition 1 of the class $\mathcal{A}^{d}$ or

$$
H^{d}(E(x, r)) \geq c r^{d}
$$

where the constant $c>0$ is independent of $x \in A$ and $r>0$ small enough. Then, for any s-extremal configuration $\omega_{N}^{*}$ on $A$,

$$
\rho\left(A, \omega_{N}^{*}\right) \leq C N^{-1 / d} .
$$

In [7], the authors removed the measure condition (3.2) and proved

Theorem 5 Let $A \in \mathcal{A}^{d}, s>d$, and let $\omega_{N}^{*}$ be an s-extremal configuration on $A$. Then

$$
\rho\left(A, \omega_{N}^{*}\right) \leq C N^{-1 / d} .
$$

Regarding point energies of s-extremal Riesz configurations, we now define a subset $\widetilde{\mathcal{A}}^{d}$ of $\mathcal{A}^{d}$ (see [7]), for which one can obtain two-sided estimates. 
Definition 6 We say that a set $A \in \widetilde{\mathcal{A}}^{d}$ if

(1) $A \in \mathcal{A}^{d}$ and

(2) there is a constant $c>0$ such that, for any $x \in A$ and $r>0$ small enough,

$$
\operatorname{diam}(E(x, r)) \geq c r .
$$

Along with trivial examples, such as a continuum or a set consisting of a finite number connected components (not singletons), the diameter condition holds for many sets consisting of infinitely many connected components. For example, Cantor sets (known to be totally disconnected) with positive Hausdorff measure are in the class $\widetilde{\mathcal{A}}^{d}$.

Theorem 7 Let $A \in \widetilde{\mathcal{A}}^{d}$ and $s>d$. Then

$$
c \leq N^{1 / d} \delta\left(A, \omega_{N}^{*}\right) \leq C
$$

and, therefore, for any $1 \leq j \leq N$,

$$
E_{j, s}\left(A, \omega_{N}^{*}\right) \geq c N^{s / d}
$$

Combining Theorems 2 and 7 yields

Corollary 8 For $s>d$ and any $s$-extremal configuration $\omega_{N}^{*}$ on $A \in \widetilde{\mathcal{A}}^{d}$,

$$
c \leq \frac{\max _{1 \leq j \leq N} E_{j, s}\left(A, \omega_{N}^{*}\right)}{\min _{1 \leq j \leq N} E_{j, s}\left(A, \omega_{N}^{*}\right)} \leq C .
$$

Thus, Corollary 8 says that, for $A \in \widetilde{\mathcal{A}}^{d}$ and $s>d$, the point energies in $s$-extremal configurations are asymptotically of the same order, as $N \rightarrow \infty$.

We note that estimates given in Theorem 7 and Corollary 8 were obtained in [6], but with the diameter condition (3.3) replaced by the measure condition (3.2), with the latter being more restrictive.

Most likely, (3.6) is the best possible assertion in the sense that the point energies are not, in general, asymptotically equal, as $N \rightarrow \infty$. (Compare with the case of the unit sphere $S^{d}$ and $0<s<d-1$ in Theorem 9(c) below.)

Simple examples show that the estimates (3.4), (3.5), and (3.6) are not valid, in general, for a set $A \in \mathcal{A}^{d}$ without an additional condition on its geometry. Indeed, as a counterexample, for $x \in \mathbb{R}^{d+1}$ with $|x|>1$, let $A=S^{d} \cup\{x\}$.

\subsection{The case $0<s<d-1$ for $S^{d}$}

In doing quadrature, it is important to know some specific properties of low discrepancy configurations, such as the separation radius, mesh ratio, and point energies. In [6], the authors established lower estimates on the separation radius for $s$-extremal Riesz configurations on $S^{d}$ for $0<s<d-1$ and proved the asymptotic equivalence of the point energies, as $N \rightarrow \infty$.

Theorem 9 Let $\omega_{N}^{*}$ be an s-extremal configuration on $S^{d}$. Then

(a) for $d \geq 2$ and $s<d-1$,

$$
\delta\left(S^{d}, \omega_{N}^{*}\right) \geq c N^{-1 /(s+1)}
$$


(b) for $d \geq 3$ and $s \leq d-2$,

$$
\delta\left(S^{d}, \omega_{N}^{*}\right) \geq c N^{-1 /(s+2)},
$$

which is sharp in $s$ for $s=d-2$;

(c) for any $0<s<d-1$,

$$
\lim _{N \rightarrow \infty} \frac{\max _{1 \leq j \leq N} E_{j, s}\left(S^{d}, \omega_{N}^{*}\right)}{\min _{1 \leq j \leq N} E_{j, s}\left(S^{d}, \omega_{N}^{*}\right)}=1 .
$$

We remark that numerical computations for a sphere (see $[4,11])$ show that, for any $s>0$, the point energies are nearly equal for almost all points that are of so-called "hexagonal" type. However, some ("pentagonal") points have elevated energies and some ("heptagonal") points have low energies. The transition from points that are "hexagonal" to those that are "pentagonal" and "heptagonal" induce scar defects, which are conjectured to vanish, as $N \rightarrow \infty$. Thus, Theorem 9 (c) provides strong evidence for this conjecture for $0<s<d-1$.

We also refer the reader to the recent paper [13], where sharp separations results for $s$-extremal configurations on the unit sphere $S^{d}$ are obtained in the case $d-1<s<d$.

\section{Discrepancy and Errors of Numerical Integration on Spheres.}

The following discrepancy and numerical type integration theorems were established in [5].

Definition 10 Let, for $\delta_{0}>0, g(t):\left[-1-\delta_{0}, 1\right) \rightarrow \mathbb{R}$ be a continuous function. We say that $g(t)$ is "admissible" if it satisfies the following conditions:

(a) $g(t)$ is strictly increasing with

$$
\lim _{t \rightarrow 1^{-}} g(t)=\infty .
$$

(b) If $g(t-\delta)$ is given by its ultraspherical expansion

$$
\sum_{n=0}^{\infty} a_{n}(\delta) P_{n}^{(d)}(t),
$$

which is valid for $t \in[-1,1]$, then we assume that, for all $n \geq 1$ and $0<\delta \leq \delta_{0}, a_{n}(\delta)>0$.

(c) The integral

$$
\int_{-1}^{1} g(t)\left(1-t^{2}\right)^{(d / 2)-1} d t
$$

converges.

One immediately checks that the following choices of admissible functions $g(t)$ yield the classical energy functionals:

$$
g_{L}^{0}(t):=\frac{1}{2} \log \frac{1}{1-t}-\frac{1}{2} \log 2,
$$

for the logarithmic energy and

$$
g_{R}^{s}(t):=\frac{1}{2^{s / 2}(1-t)^{s / 2}}, \quad s>0,
$$

for the Riesz s-energy. 
For a set $\omega_{N}=\left\{x_{1}, \ldots, x_{N}\right\}$ of points on $S^{d}$, similarly to (2.2) and (2.3), we set

$$
E_{g}\left(S^{d}, \omega_{N}\right)=\sum_{1 \leq i<j \leq N}^{N} g\left(<x_{i}, x_{j}>\right)
$$

where $<\cdot>$ denotes inner product in $\mathbb{R}^{d+1}$, and define

$$
\mathcal{E}_{g}\left(S^{d}, N\right)=\min _{\omega_{N} \subset S^{d}} E_{g}\left(S^{d}, \omega_{N}\right) .
$$

A point set $\omega_{N}^{*}$, for which the minimal energy $\mathcal{E}_{g}\left(S^{d}, N\right)$ is attained, is called a minimal g-energy point set. It is clear that any rotation of a point set of minimal energy again gives a point set of minimal energy. It was shown in [5] that, for any admissible function $g(t)$, the energy integral

$$
I_{g}\left(S^{d}, \nu\right):=\iint_{S^{d} \times S^{d}} g(<x, y>) d \nu(x) d \nu(y)
$$

is minimized by the normalized area measure $\sigma_{d}$ amoungst all Borel probability measures $\nu$ on $S^{d}$. Using arguments similar to those in Examples 1 and 2, one expects that the normalized counting measure $\nu^{\omega_{N}^{*}}$ of points in $\omega_{N}^{*}$ gives a discrete approximation to the normalized area measure $\sigma_{d}$ in the sense that the integral of any continuous function $f$ on $S^{d}$ against $\sigma_{d}$ is approximated by the $\left(N^{-1}\right)$-weighted discrete sum over the points in $\omega_{N}^{*}$.

Theorem 11 (g-energy and discrepancy) Let $g(t)$ be admissible, $d \geq 2, \omega_{N}$ be a collection of $N$ points on $S^{d}$, $f$ be a polynomial of degree at most $n \geq 1$ on $\mathbb{R}^{d+1}$, and $0<\delta \leq \delta_{0}$. Then

(a) $\left|R\left(f, \omega_{N}\right)\right| \leq\|f\|_{2}\left(\frac{2 N^{-2} E_{g}\left(S^{d}, \omega_{N}\right)-a_{0}(\delta)+N^{-1} g(1-\delta)}{\min _{1 \leq k \leq n}\left[a_{k}(\delta) / Z(d, k)\right]}\right)^{1 / 2}$

with $Z(d, k)$ counting the linearly independent spherical harmonics of degree $k$ on $S^{d}$. Moreover, if $q=q(d)$ is the smallest integer satisfying $2 q \geq d+3$, then there exists a positive constant $C$, independent of $N$ and $\omega_{N}$, such that uniformly on $m \geq 1$ and $0<\delta<\delta_{0}$ there holds

$$
D_{N}\left(\omega_{N}\right) \leq C\left\{\frac{1}{m}+\left(\frac{2 N^{-2} E_{g}\left(S^{d}, \omega_{N}\right)-a_{0}(\delta)+N^{-1} g(1-\delta)}{\min _{1 \leq k \leq n}\left[a_{k}(\delta) / Z(d, k)\right]}\right)^{1 / 2}\right\} .
$$

(b) Let $f$ be a continuous function on $S^{d}$ satisfying

$$
|f(x)-f(y)| \leq C_{f} \arccos (\langle x, y\rangle), \quad x, y \in S^{d} .
$$

Then, for any $n \geq 1$,

$$
\left|R\left(f, \omega_{N}\right)\right| \leq 12 C_{f} \frac{d}{n}+\left(\frac{2 N^{-2} E_{g}\left(S^{d}, \omega_{N}\right)-a_{0}(\delta)+N^{-1} g(1-\delta)}{\min _{1 \leq k \leq n}\left[a_{k}(\delta) / Z(d, k)\right]}\right)^{1 / 2} .
$$

Remark 12 (a) Theorem 11 shows that second order terms in the expansion of minimal energies determine rates in errors of numerical integration over spheres. Indeed, in general, one hopes that the energy term $2 N^{-2} E_{g}\left(S^{d}, \omega_{N}\right)$ and the leading term $a_{0}(\delta)$ cancel each other sufficiently to allow for an exact error. An application of this idea was exploited first in [5] in the case $s=d$. Here, the energy integral diverges for every probability measure supported on $S^{d}$ and the rate of convergence given in Theorem 11 is $\sqrt{\log \log n / \log n}$. (See Theorem 13 below.) 
(b) Brauchart [1] has considered invariance principles for energy functionals based on Theorem 11, which allow for a family of $g$-functionals to conclude, in particular, that for any $s$-extremal configuration on $S^{d}, 0<s<d$, the error term in Theorem 11 goes to zero, as $N \rightarrow \infty$, and, moreover, this error can be explicitly calculated due to the finiteness of the corresponding energy integral.

(c) The fact that the error in Theorem 11 goes to zero for any $s$-extremal configuration (without rate of convergence) follows from the finiteness of the energy integral $(0<s<d)$, Theorem 13 below $(s=d)$, and results of [9] and [10] $(s \geq d)$.

We now quantify the error in Theorem 11 for $d$-extremal configurations on $S^{d}$ (which are sets of minimal $g_{R}^{d}$-energy).

Theorem 13 Let $f$ be a continuous function on $S^{d}$ satisfying (4.1), and let $\omega_{N}^{*}$ be a d-extremal configuration. Then

$$
\left|R\left(f, \omega_{N}^{*}\right)\right|=\mathcal{O}\left(\frac{C_{f}+\|f\|_{\infty} \sqrt{\log \log N}}{\sqrt{\log N}}\right)
$$

with the implied constant depending only on d. Moreover,

$$
D\left(\omega_{N}^{*}\right)=\mathcal{O}\left(\sqrt{\frac{\log \log N}{\log N}}\right) .
$$

We remark that it is widely believed that the order above may indeed be improvable to a negative power of $N$. Thus far, however, it is not clear how to prove whether this belief is indeed correct.

Acknowledgement This paper is based on the talk given by the first author at the International Conference on "Algorithms for Approximation V", University of Leicester, England, July 18-23, 2005.

\section{References}

[1] J. Brauchart, Discrepancy estimates for Riesz points on the sphere, Manuscript.

[2] B. Bajnok, S. B. Damelin, J. Li and G. Mullen, A constructive finite field method for scattering points on the surface of a d-dimensional sphere, Computing 68 (2002): 97-109.

[3] S. V. Borodachov, D. P. Hardin, and E. B. Saff, Asymptotics for discrete weighted minimal Riesz energy problems on rectifiable sets, submitted.

[4] M. Bowick, A. Cacciuto, D. R. Nelson, and A. Travesset, Crystalline order on a sphere and the generalized Thomson problem, Phys. Rev. Lett., 89 (2002): 185502.

[5] S. B. Damelin and P. Grabner, Energy functionals, numerical integration and asymptotic equidistribution on the sphere, J. Complexity, 19 (2003): 231-246; Corrigendum, to appear, J. Complexity.

[6] S. B. Damelin and V. Maymeskul, On point energies, separation radius and mesh norm for s-extremal configurations on compact sets in $\mathbb{R}^{n}$, to appear, Journal of Complexity.

[7] S. B. Damelin and V. Maymeskul, On point energies, separation radius and mesh norm for s-extremal configurations on compact sets in $\mathbb{R}^{n}$, II, submitted to Geometric Analysis.

[8] P. Dragnev, D. A. Legg and D. W. Townsend, On the separation of logarithmic points on the sphere, Approximation Theory X: Abstract and Classical Analysis, Charles K. Chui, Larry L. Schumaker, and Joachim Stoeckler (eds.), Vanderbilt University Press (2002): 137-144. 
[9] M. Götz and E. B. Saff, Note on d-extremal configurations for the sphere in $\mathbb{R}^{d+1}$, In: Recent progress in multivariate approximation, Internat. Ser. Numer. Math., Birkhauser, Basel, 137 (2001): 159-162.

[10] D. Hardin and E. B. Saff, Minimal Riesz energy point configurations for rectifiable d-dimensional manifolds, Advances in Mathematics, 193 (2005): 174-204.

[11] D. Hardin and E. B. Saff, Discretizing Manifolds via Minimum Energy Points, Notices of the AMS (Nov. 2004): 1186-1194.

[12] A. B. J. Kuijlaars and E. B. Saff, Asymptotics of minimal discrete energy on the sphere, Trans. Amer. Math. Soc., 350 (1998): 523-538.

[13] A. B. J. Kuijlaars, E. B. Saff, and X. Sun, On separation of minimal Riesz energy points on spheres in Euclidean spaces, submitted.

[14] A. Lubotzky, R. Phillips, and P. Sarnak, Hecke operators and distributing points on the sphere I, Comm. Pure App. Math. 39 (1986): S148-S186.

[15] A. Lubotzky, R. Phillips, and P. Sarnak, Hecke operators and distributing points on the sphere II, Comm. Pure App. Math. 40 (1987): 401-420.

[16] A. Martinez-Finkelshtein, V. V. Maymeskul, E. A. Rakhmanov, and E. B. Saff, Asymptotics for minimal discrete Riesz energy on curves in $\mathbb{R}^{d}$, Canad. J. Math. 56 (2004): 529-552.

[17] E. A. Rakhmanov, E. B. Saff, and Y. M. Zhou, Minimal discrete energy on the sphere, Mathematical Research Letters, 1 (1994): 647-662.

S.B. Damelin

Institute for Mathematics and its Applications

University of Minnesota

400 Lind Hall, 207 Church Hill, S.E

Minneapolis, MN 55455, U.S.A.

Email: damelin@ima.umn.edu

V. Maymeskul

Department of Mathematical Sciences

Georgia Southern University

P.O. Box 8093

Statesboro, Georgia 30460, U.S.A.

Email: vmaymesk@georgiasouthern.edu 